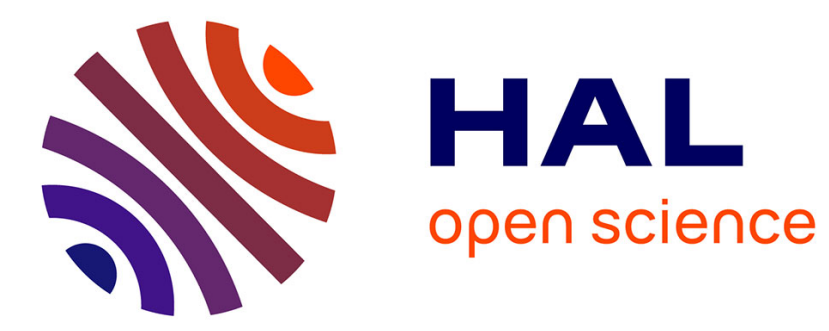

\title{
Explicit estimates on the summatory functions of the Möbius function with coprimality restrictions
}

\author{
Olivier Ramaré
}

\section{To cite this version:}

Olivier Ramaré. Explicit estimates on the summatory functions of the Möbius function with coprimality restrictions. Acta Arithmetica, 2014, 165 (1), pp.1-10. 10.4064/aa165-1-1 . hal-02572851

\section{HAL Id: hal-02572851 \\ https://hal.science/hal-02572851}

Submitted on 13 May 2020

HAL is a multi-disciplinary open access archive for the deposit and dissemination of scientific research documents, whether they are published or not. The documents may come from teaching and research institutions in France or abroad, or from public or private research centers.
L'archive ouverte pluridisciplinaire HAL, est destinée au dépôt et à la diffusion de documents scientifiques de niveau recherche, publiés ou non, émanant des établissements d'enseignement et de recherche français ou étrangers, des laboratoires publics ou privés. 


\title{
Explicit estimates on the summatory functions of the Moebius function with coprimality restrictions
}

\author{
Olivier Ramaré, \\ CNRS, Laboratoire Paul Painlevé, \\ Université Lille 1, \\ 59655 Villeneuve d'Ascq, France \\ Email: ramare@math.univ-lille1.fr
}

October 9, 2012

\begin{abstract}
We prove that $\left|\sum_{\left\{\begin{array}{c}d \leq x, \\ (d, q)=1\end{array}\right.} \mu(d) / d\right| \leq 2(q / \varphi(q)) / \log (x / q)$ for every $x>q \geq 1$ and similar estimates for the Liouville functions. We give also better constants when $x / q$ is larger.
\end{abstract}

\section{Introduction}

In explicit analytic number theory, one needs very often to evaluate the average of a multiplicative function, say $f$. The usual strategy is to compare this function to a more usual model $f_{0}$, as in [12, Lemma 3.1]. This process is also well detailed in [2]. When the model is $f_{0}=1$, the situation is readily cleared out; it is also well studied when this model is the divisor function in [1, Corollary 2.2]. We signal here that the case of the characteristic function of the squarefree numbers is specifically handled in [4]. The next problem is to use the Moebius function as a model. In this case the necessary material can be found in [13], though of course the saving is much less and may be insufficient: when the model is 1 or the divisor function, or the characteristic

2010 Mathematics Subject Classification: Primary 11N37, 11Y35; Secondary 11A25. Key words and phrases: Explicit estimates, Moebius function. 
function of the squarefree integers, the saving is a power of the size of the variable, while now it is only a logarithm (or the square of one according to whether one says that the trivial estimate for $\sum_{d \leq D} \mu(d) / d$ is 1 or $\left.\log D\right)$. One of the consequences is that one has to be more careful, and thrifty, when it comes to small variations. The variations we consider here is the addition of a coprimality condition $(d, q)=1$, for some fixed $q$, on the ranging variable $d$. Our first aim is thus to show how to get explicit estimates for the family of functions

$$
m_{q}(x)=\sum_{\substack{n \leq x,(n, q)=1}} \mu(n) / n, \quad m(x)=m_{1}(x)
$$

from explicit estimates concerning solely $m(x)$. The definition of the Liouville function $\lambda(n)$ is recalled below in (1.3), while the auxiliary function $\ell_{q}$ is defined in (1.4).

Theorem 1.1. We have, when $1 \leq q<x$, where $q$ is an integer and $x$ a real number,

$$
\left|\sum_{\substack{n \leq x,(n, q)=1}} \frac{\mu(n)}{n}\right| \leq \frac{q}{\varphi(q)} \frac{2}{\log (x / q)}, \quad\left|\sum_{\substack{n \leq x,(n, q)=1}} \frac{\lambda(n)}{n}\right| \leq \frac{q}{\varphi(q)} \frac{0.55}{\log (x / q)}
$$

Moreover $\log (x / q)\left|\ell_{q}(x)\right| \leq 0.155 \frac{q}{\varphi(q)}$ and $\log (x / q)\left|m_{q}(x)\right| \leq \frac{8}{7} \frac{q}{\varphi(q)}$ when $x / q \geq 3310$. We also have $\log (x / q)\left|m_{q}(x)\right| \leq \frac{5}{7} \frac{q}{\varphi(q)}$ when $x / q \geq 9960$.

The sole previous estimate on $m_{q}(x)$ seems to be [7, Lemma 10.2] which bounds $\left|m_{q}(x)\right|$ uniformly by 1 . The estimate for $m(x)$ that will provide the initial step comes from [13]

$$
|m(x)| \leq 0.03 / \log x \quad\left(x \geq X_{0}=11815\right) .
$$

Let us first note that the simplest treatment of this condition via the Moebius function, i.e. writing

$$
\mathbb{1}_{(d, q)=1}=\sum_{\substack{\delta|q, \delta| d}} \mu(\delta)
$$

does not work here. Indeed we get:

$$
\sum_{\substack{d \leq D,(d, q)=1}} \frac{\mu(d)}{d}=\sum_{\delta \mid q} \sum_{\delta \mid d \leq D} \frac{\mu(d)}{d}=\sum_{\delta \mid q} \frac{\mu(\delta)}{\delta} \sum_{\substack{d \leq D / \delta,(d, \delta)=1}} \frac{\mu(d)}{d}
$$


and we are back to the initial problem with different parameters. The classical workaround (used for instance in [10, near (7)] but already known by Landau) runs as follows: we determine a function $g_{q}$ such that $\mathbb{1}_{(n, q)=1} \mu(n)=$ $g_{q} \star \mu(n)$, where $\star$ denotes the arithmetic convolution product. The drawback of this method is that the support of $g$ is not bounded (determining $g_{q}$ by comparing the Dirichlet series is a simple exercise). So if we write

$$
\sum_{\substack{d \leq D,(d, q)=1}} \mu(d) / d=\sum_{\delta \leq D} g_{q}(\delta) \sum_{d \leq D / \delta} \mu(d) / d,
$$

we are forced to two things:

1. using estimates for $\sum_{d \leq D / \delta} \mu(d) / d$ when $D / \delta$ can be small,

2. completing the sum over $\delta$ to get a decent result.

Both steps introduce quite a loss when $q$ is not specified. We propose here a different approach by introducing the Liouville function as an intermediary. This function $\lambda(n)$ is the completely multiplicative function that is 1 on integers that have an even number of prime factors - counted with multiplicity - and -1 otherwise. It satisfies

$$
\sum_{n \geq 1} \frac{\lambda(n)}{n^{s}}=\frac{\zeta(2 s)}{\zeta(s)} .
$$

We introduce the family of auxiliary functions

$$
\ell_{q}(x)=\sum_{\substack{n \leq x,(n, q)=1}} \lambda(n) / n, \quad \ell(x)=\ell_{1}(x) .
$$

Our process runs as follows: we derive bounds for $\ell(x)$ from bounds on $m(x)$ and some computations, derive bounds on $\ell_{q}(x)$ from bounds on $\ell(x)$, and finally derive bounds on $\mu_{q}(x)$ from bounds on $\ell_{q}(x)$. The theoretical steps are contained in the three Lemmas 2.3, 2.5 and 3.2.

We complete this introduction by signalling that [14] contains explicit estimates with a large range of uniformity for sums of the shape

$$
\sum_{\substack{d \leq x \\(d, r)=1}} \frac{\mu(d)}{d^{1+\varepsilon}}
$$

and for a similar sum but with the summand $\mu(d) \log (x / d) / d^{1+\varepsilon}$. The path we followed there is essentially elementary and the saving is less.

I thank Harald Helfgott for interesting discussions that pushed me into pulling this note out of its drawer, as well as the referee for his/her careful reading that has helped getting a better version of this note. 


\section{From the Moebius function to the Liou- ville function}

Lemma 2.1. For $2 \leq x \leq 906000000$, we have $|\ell(x)| \leq 1.347 / \sqrt{x}$.

For $2 \leq x \leq 1.1 \cdot 10^{10}$, we have $|\ell(x)| \leq 1.41 / \sqrt{x}$.

For $1 \leq x \leq 1.1 \cdot 10^{10}$, we have $|\ell(x)| \leq \sqrt{2 / x}$.

The computations have been run with PARI/GP (see [11]), speeded by using gp2c as described for instance in [1]. We mention here that [6] proposes an algorithm to compute isolated values of $M(x)$. This can most probably be adapted to compute isolated values of $\ell(x)$, but does not seem to offer any improvement for bounding $|\ell(x)|$ on a large range. In [3], the authors show that

$$
\ell(x) \geq 0, \quad(x \leq 72185376951205)
$$

and that

$$
\ell(x) \geq-2.0757640 \times 10^{-9}, \quad(x \leq 75000000000000)
$$

This takes care of the lower bound for $\ell(x)$. The computations we ran are much less demanding in time and algorithm, but however rely on a large enough sieve-kind of table to compute values of $\lambda(n)$ on some very large range. Harald Helfgott (indirectly) pointed out to me that the RAMmemory can be very large nowadays, allowing to precompute large quantities to which one has an almost immediate access. Here is a simplified version of the main loop:

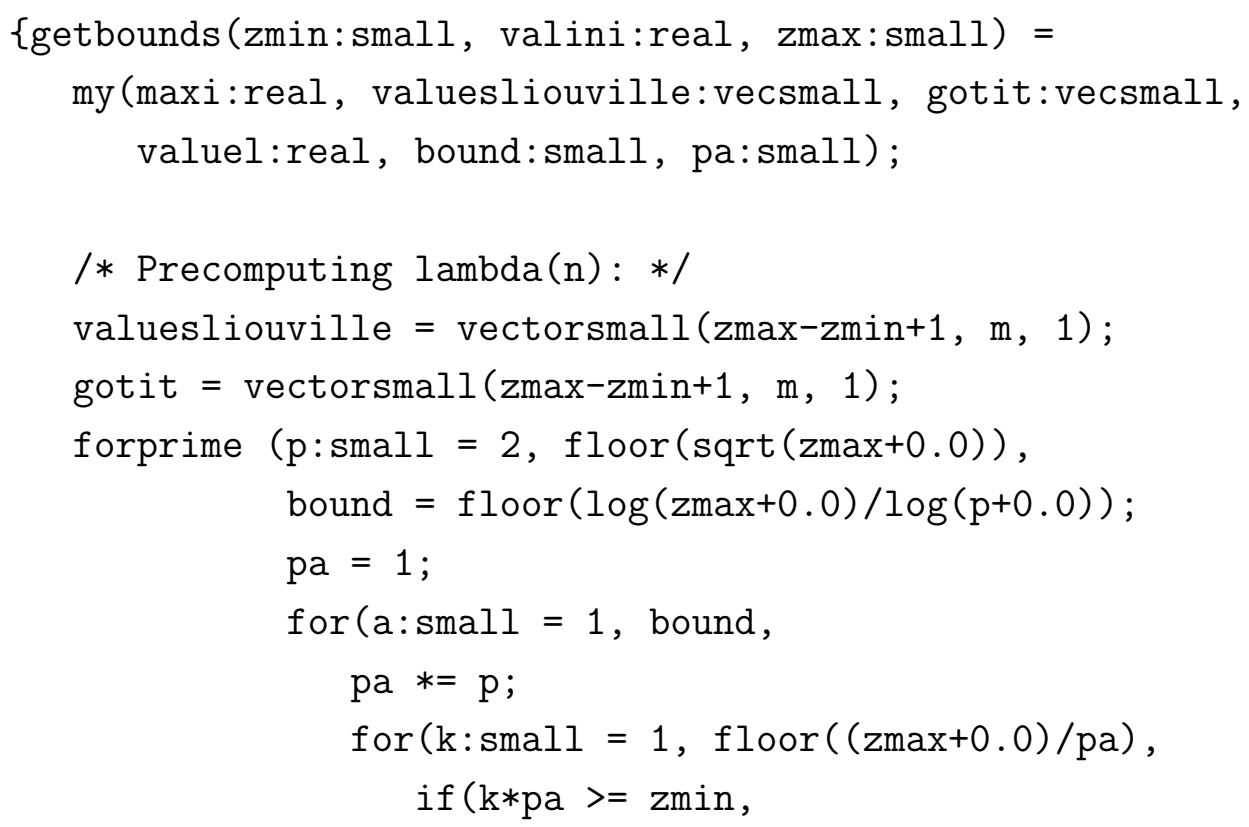




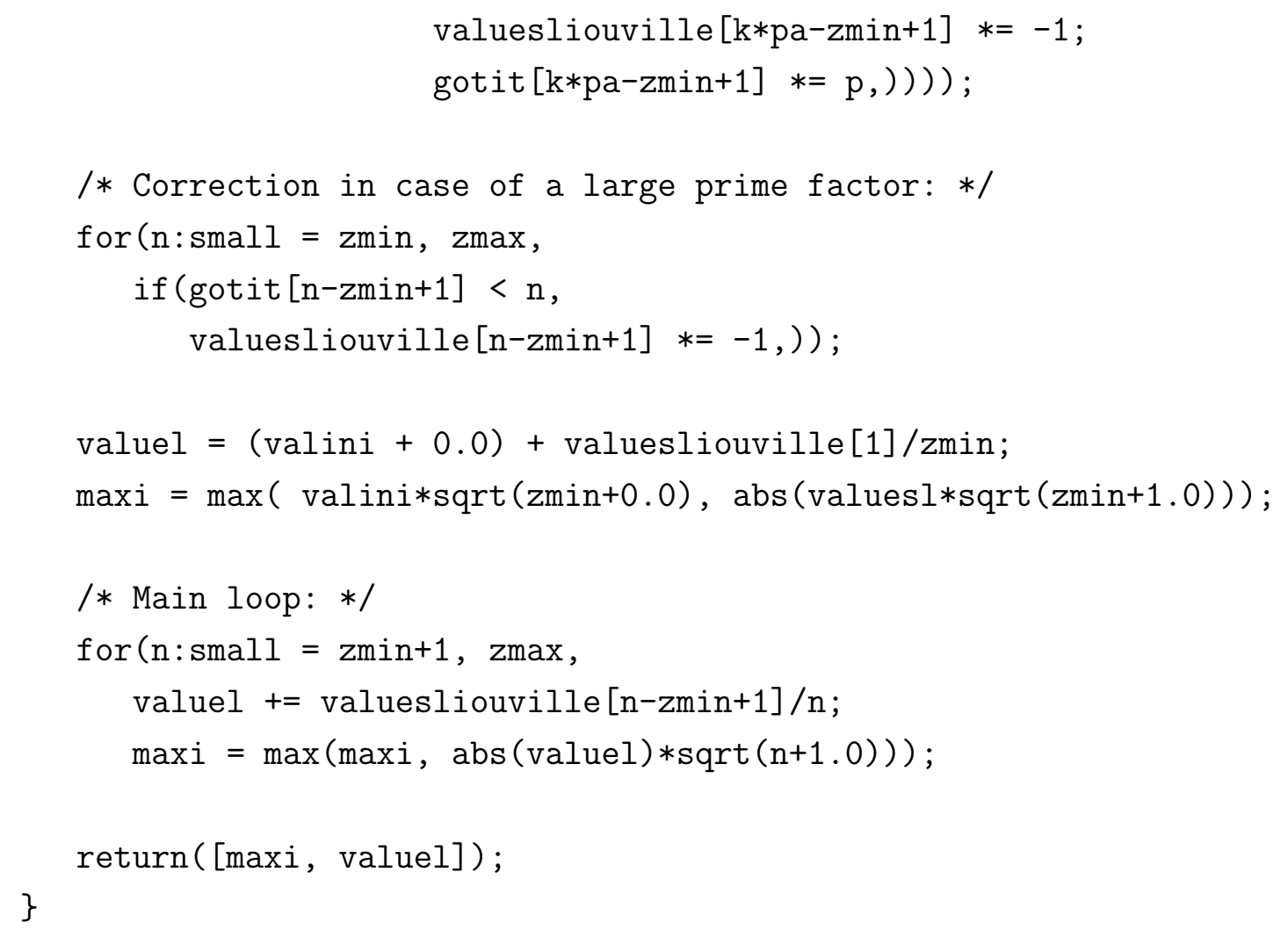

We used this loop to compute our maximum on intervals of length $2 \cdot 10^{7}$. The main function aggregates these results by making the interval vary. The computations took about half a day on a 64 bits fast desktop equipped of $8 \mathrm{G}$ of RAM. In the actual script, we also checked that the computed value of $\ell(x)$ is non-negative in this range. Going farther would improve on the final constants, but only when $x / q$ is large. We compared $|\ell(x)|$ with $1 / \sqrt{x}$, and this seems correct for small values, but the works [9] and [8] suggest that the maximal order is larger than that.

Lemma 2.2. The function

$$
T(y): y \mapsto \frac{\log y}{y} \int_{\sqrt{X_{0}}}^{y} \frac{d v}{\log v}
$$

satisfies $T\left(10^{5}\right) \leq 1.119$.

Proof. We check numerically that the function $T$ is increasing and then decreasing, with a maximum around 12478.8 with value $1.118598+\mathcal{O}^{*}\left(10^{-6}\right)$. But this is only an observation, since a computer computes only a sample of values. The bounded of $T$ being easily bounded, we can rigorously infer the claimed upper bound. The proof of Theorem 1.1 contains page 9 a similar discussion, though with somewhat more details. The reader may also consult [5] where a similar process is fully detailled. 
The following lemma is a simple exercise:

Lemma 2.3. We have

$$
\ell_{q}(x)=\sum_{\substack{u^{2} \leq x,(u, q)=1}} m_{q}\left(x / u^{2}\right) / u^{2}
$$

We shall use it only when $q=1$, but it is equally easy to state it in general.

Lemma 2.4. For $x>1$, we have $|\ell(x)| \leq 0.55 / \log x$.

For $x \geq 3310$, we have $|\ell(x)| \leq 0.155 / \log x$.

For $x \geq 8918$, we have $|\ell(x)| \leq 0.099 / \log x$.

Proof. We appeal to Lemma 2.3 (with $q=1$ ) and separate the sum according to $u \leq U$ or $u>U$ where $x / U^{2} \geq X_{0}$. When $u \leq U$ we apply (1.2), in the other case we use that $|m(x)| \leq 1$

$$
|\ell(x)| \leq 0.03 \sum_{u \leq U} \frac{1}{u^{2} \log \left(x / u^{2}\right)}+\frac{1+U^{-1}}{U}
$$

With $f(t)=1 /\left(t^{2} \log \left(x / t^{2}\right)\right)$, we check that

$$
f^{\prime}(t)=-\frac{2}{u^{3} \log \left(x / t^{2}\right)}+\frac{2}{u^{3} \log ^{2}\left(x / t^{2}\right)} .
$$

This quantity is non-positive $1 \leq t \leq U$, since then $x / U^{2} \geq X_{0} \geq e$. We thus have

$$
\begin{aligned}
\sum_{u \leq U} \frac{1}{u^{2} \log \left(x / u^{2}\right)} & =\sum_{u \leq U}\left(f(U)-\int_{1}^{U} f^{\prime}(t) d t\right) \leq U f(U)-\int_{1}^{U} t f^{\prime}(t) d t \\
& \leq f(1)+\int_{1}^{U} f(t) d t \leq \frac{1}{\log x}+\int_{1}^{U} \frac{d t}{t^{2} \log \left(x / t^{2}\right)}
\end{aligned}
$$

Changing variables we get

$$
\sum_{u \leq U} \frac{1}{u^{2} \log \left(x / u^{2}\right)} \leq \frac{1}{\log x}+\frac{1}{\sqrt{x}} \int_{\sqrt{x / U^{2}}}^{\sqrt{x}} \frac{d v}{2 \log v}
$$

It follows that

$$
|\ell(x)| \leq \frac{0.03}{\log x}+\frac{0.03}{\sqrt{x}} \int_{\sqrt{X_{0}}}^{\sqrt{x}} \frac{d v}{2 \log v}+\frac{1+\sqrt{X_{0} / x}}{\sqrt{x / X_{0}}} .
$$


We employ Lemma 2.2 at this level. Hence, when $x \geq 10^{10}$,

$$
\begin{aligned}
|\ell(x)| & \leq \frac{0.03}{\log x}+\frac{0.03 \cdot 2 \cdot 1.119}{\log x}+\frac{1+\sqrt{X_{0} / x}}{\sqrt{x / X_{0}}} \\
& \leq \frac{0.0983}{\log x} \leq \frac{0.099}{\log x} .
\end{aligned}
$$

We extend it to $x \geq 18033$ via Lemma 2.1, part one and two, and to $x \geq$ 8918 by direct inspection. This inequality extends to $x \geq 1$ by weakening the constant 0.099 to 0.55 . It is straighforward to use some mild computations to check the validity of the bound 0.155 when $x \geq 3310$.

\section{Adding coprimality conditions}

Our tool is provided by the simple elementary lemma.

Lemma 2.5. We have

$$
\ell_{q}(x)=\sum_{d \mid q} \frac{\mu^{2}(d)}{d} \ell(x / d)
$$

The first part of Theorem 1.1 follows immediately by combining Lemma 2.5 together with Lemma 2.4. Actually, what comes out is the bound

$$
\left|\ell_{q}(x)\right| \leq \frac{0.55}{\log (x / q)} \sum_{d \mid q} \frac{\mu^{2}(d)}{d}=\frac{0.55}{\log (x / q)} \prod_{p \mid q} \frac{p+1}{p} .
$$

As the function $q / \varphi(q)$ is easier to remember and $\prod_{p \mid q} \frac{p+1}{p} \leq q / \varphi(q)$, we simplify the above into

$$
\left|\ell_{q}(x)\right| \leq \frac{0.55}{\log (x / q)} \frac{q}{\varphi(q)} .
$$

When $x / q \geq 3310$, one can replace 0.55 by 0.155 , and when $x / q \geq 8918$, by $1 / 10$.

\section{Back to the Moebius function with copri- mality coditions}

Let us start with a wide ranging estimate:

Lemma 3.1. We have, for every integer $q \geq 1$ and every real number $x \geq 1$, $\left|\ell_{q}(x)\right| \leq \pi^{2} / 6$. 
Proof. This a direct consequence of Lemma 2.3 and [7, Lemma 10.2]. ${ }^{1}$

The following lemma is again a simple exercise.

Lemma 3.2. We have

$$
m_{q}(x)=\sum_{\substack{u^{2} \leq x,(u, q)=1}} \frac{\mu(u)}{u^{2}} \ell_{q}\left(x / u^{2}\right) .
$$

Proof of Theorem 1.1. We proceed to prove the estimate concerning $m_{q}(x)$, starting by appealing to Lemma 3.2. We have, for a real parameter $U$ such that $x>U^{2} q$,

$$
\begin{aligned}
\left|m_{q}(x)\right| & \leq \sum_{u^{2} \leq x} \frac{\mu^{2}(u)}{u^{2}}\left|\ell_{q}\left(x / u^{2}\right)\right| \\
& \leq \sum_{u \leq U} \frac{q}{\varphi(q)} \frac{0.55 \mu^{2}(u)}{u^{2} \log \left(x /\left(u^{2} q\right)\right)}+\frac{\pi^{2}}{6} \sum_{u>U} \frac{\mu^{2}(u)}{u^{2}} \\
& \leq \frac{q}{\varphi(q) \log (x / q)}\left(\sum_{u \leq U} \frac{0.55 \mu^{2}(u)}{u^{2}\left(1-\frac{2 \log u}{\log (x / q)}\right)}+\frac{\pi^{2}}{6} \sum_{u>U} \frac{\mu^{2}(u)}{u^{2}} \log (x / q)\right) .
\end{aligned}
$$

This is our starting inequality.

Small values of $x^{*}=x / q$

We first notice that the bound provided by [7, Lemma 10.2] proves the estimate $\left|m_{q}(x)\right| \log x^{*} \leq 2 q / \phi(q)$ when $\log x^{*} \leq 2$.

We define

$$
\rho(U, y)=0.55 \sum_{u \leq U} \frac{\mu^{2}(u)}{u^{2}\left(1-\frac{2 \log u}{y}\right)}+\frac{\pi^{2}}{6} \sum_{u>U} \frac{\mu^{2}(u)}{u^{2}} y .
$$

Note that $\rho(U, y)=\rho([U], y)$ where $[U]$ is the integer part of $U$. We want to determine an upper bound for

$$
\min _{1 \leq U<\exp (y / 2)} \rho(U, y)
$$

This will determine our choice of parameter $U$. Here is the GP/Pari (see [11]) script that we have used:

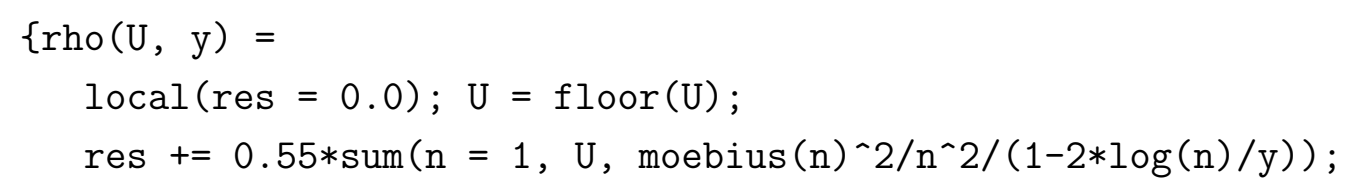

\footnotetext{
${ }^{1}$ If we were to adapt the proof presented in [7] to the case of $\lambda$ instead of $\mu$, we would reach the bound 2 and not $\pi^{2} / 6$.
} 


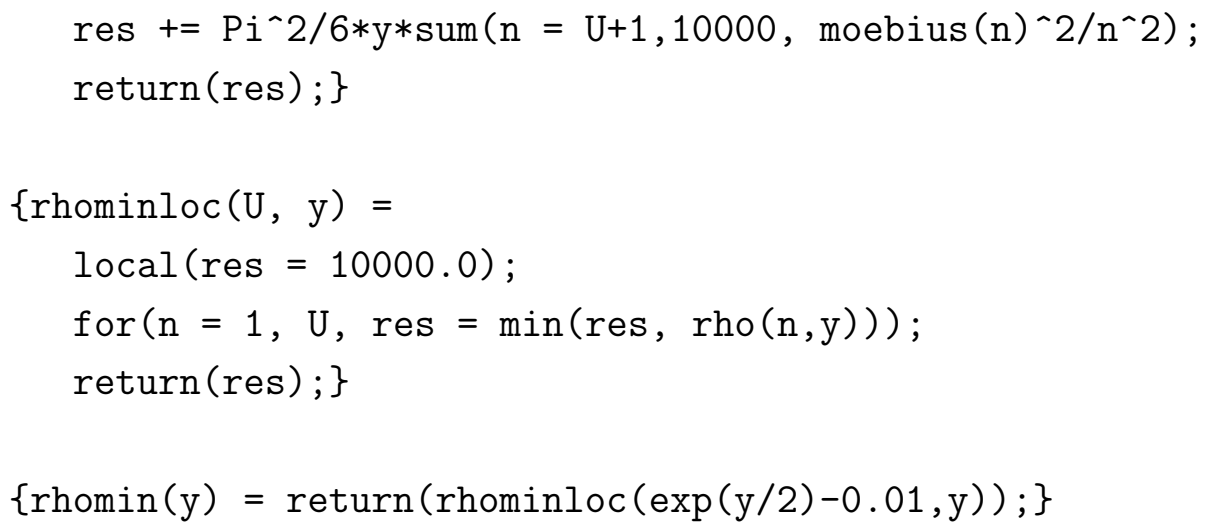

We use this part for $y=\log x \leq 8$. We get a numerical maximum around $y=1.72$ with value $\leq 2.0196 \cdots$. When $2 \leq y \leq 3$, we get a numerical local maximum around $y=2.5315$ with value $1.9749983 \cdots$. Other numerical local maxima appear between each integer, but the values taken there are smaller. As the referee rightly pointed out, it is better to specify really what the adjective "numerical" covers in the above description:

- We cannot claim that the function of $y$ indeed has a single maximum in a given interval, but only that this is so on a selection of values very narrowly placed (every $10^{-7}$ say).

- It is however easy to see that the derivative of $\rho(U, y)$ with respect to $y$ is bounded by $($ since $U \leq \exp (y / 2)-0.01$, we have $y-2 \log u \geq$ $\left.\left(1-e^{-0.01}\right) y / 2\right)$

$$
\frac{\partial \rho(U, y)}{\partial y}=-0.55 \sum_{u \leq U} \frac{\mu^{2}(u) \log u}{u^{2} y(y-2 \log u)}+\frac{\pi^{2}}{6} \sum_{u>U} \frac{\mu^{2}(u)}{u^{2}} \in[-160,1] .
$$

This tells us that is a specific value of $U$, say $U_{0}$ yields an upper bound for $\min _{1 \leq U<\exp (y / 2)} \rho(U, y)$ for some $y$ and this ensures that $\min _{1 \leq U<\exp (y / 2)} \rho\left(U, y^{\prime}\right)$ is not much more when $y^{\prime}$ and $y$ are close enough; more specifically, we see that the corresponding error term is taken care of by the truncation of the final result.

The article [5] contains full details of a similar process.

\section{Large values of $x^{*}=x / q$}

We start from Lemma 3.2, from which we deduce the simpler bound:

$$
\left|m_{q}(x)\right| \leq \sum_{u^{2} \leq x}\left|\ell_{q}\left(x / u^{2}\right)\right| / u^{2}
$$


which we then exploit in the same way as what is done in the proof of Lemma 2.4, replacing the bound $|m(x)| \leq 1$ by Lemma 3.1. With $x=e U^{2} q$ and $x^{*}=x / q$, we thus get

$$
\begin{aligned}
\left|m_{q}(x)\right| & \leq \frac{q}{\varphi(q)} \frac{0.55}{\log x^{*}}+\frac{0.55 q}{\varphi(q)} \int_{1}^{\sqrt{x^{*} / e}} \frac{d u}{u^{2} \log \left(x^{*} / u^{2}\right)}+\frac{\pi^{2} \sqrt{e}}{6} \frac{1+\sqrt{e} x^{*-1 / 2}}{\sqrt{x^{*}}} \\
& \leq \frac{q}{\varphi(q)} \frac{0.55}{\log x^{*}}+\frac{0.55 q}{\varphi(q) \sqrt{x^{*}}} \int_{e}^{\sqrt{x^{*}}} \frac{d v}{2 \log v}+\frac{\pi^{2} \sqrt{e}}{6} \frac{1+\sqrt{e} x^{*-1 / 2}}{\sqrt{x^{*}}} \\
& \leq c\left(x^{*}\right) \frac{q}{\varphi(q) \log x^{*}}
\end{aligned}
$$

with

$$
c\left(x^{*}\right)=0.55+0.55 \frac{\log x^{*}}{\sqrt{x^{*}}} \int_{e}^{\sqrt{x^{*}}} \frac{d v}{2 \log v}+\frac{\pi^{2} \sqrt{e}}{6} \frac{1+\sqrt{e} x^{*-1 / 2}}{\sqrt{x^{*}}} \log x^{*} .
$$

Some numerical work shows that the quantity in parentheses is $\leq 1.71$ when $x^{*} \geq 2500$. When $x^{*} \geq 3310$, we can single out the term $n=1$ and modify the coefficient 0.55 to 0.155 :

$$
\begin{aligned}
c_{1}\left(x^{*}\right)=0.155+0.55 \frac{\log x^{*}}{4 \log x^{*}}+0.55 \frac{\log x^{*}}{\sqrt{x^{*}}} & \int_{e}^{\sqrt{x^{*} / 4}} \frac{d v}{2 \log v} \\
& +\frac{\pi^{2} \sqrt{e}}{6} \frac{1+\sqrt{e} x^{*-1 / 2}}{\sqrt{x^{*}}} \log x^{*} .
\end{aligned}
$$

When $x^{*} \geq 3 \times 3310$, we single out the terms of index 1,2 , and 3 similarly. The proof of Theorem 1.1 is complete.

\section{References}

[1] D. Berkane, O. Bordellès, and O. Ramaré. Explicit upper bounds for the remainder term in the divisor problem. Math. of Comp., 81(278):1025-1051, 2012.

[2] P. Berment and O. Ramaré. Ordre moyen d'une fonction arithmétique par la méthode de convolution. Revue de Mathématique Spéciale, 212(1):1-15, 2012.

[3] P. Borwein, R. Ferguson, and M.J. Mossinghoff. Sign changes in sums of the Liouville function. Math. Comp., 77(263):1681-1694, 2008.

[4] H. Cohen, F. Dress, and M. El Marraki. Explicit estimates for summatory functions linked to the Möbius $\mu$-function. Funct. Approx. Comment. Math., 37(part 1):51-63, 2007. 
[5] J. Arias de Reyna and J. van de Lune. A proof of a trigonometric inequality. A glimpse inside the mathematical kitchen. J. Math. Inequal., 5(3):341-353, 2011.

[6] M. Deléglise and J. Rivat. Computing the summation of the Möbius function. Exp. Math., 5(4):291-295, 1996.

[7] A. Granville and O. Ramaré. Explicit bounds on exponential sums and the scarcity of squarefree binomial coefficients. Mathematika, 43(1):73107, 1996.

[8] T. Kotnik and J. van de Lune. On the order of the Mertens function. Experiment. Math., 13(4):473-481, 2004.

[9] H.L. Montgomery. Zeros of approximations to the zeta function. In Studies in pure mathematics, pages 497-506. Birkhäuser, Basel, 1983.

[10] Y. Motohashi. Primes in arithmetic progressions. Invent. Math., 44(2):163-178, 1978.

[11] The PARI Group, Bordeaux. PARI/GP, version 2.5.2, 2011. http: //pari.math.u-bordeaux.fr/.

[12] O. Ramaré. On Snirel'man's constant. Ann. Scu. Norm. Pisa, 21:645706, 1995. http://math.univ-lille1.fr/ ramare/Maths/Article. pdf.

[13] O. Ramaré. From explicit estimates for the primes to explicit estimates for the Moebius function. To appear in Acta Arith., 2012.

[14] O. Ramaré. Some elementary explicit bounds for two mollifications of the Moebius function. Submitted to Acta Arith., 2012. 